\title{
Some new fixed point theorems for compatible mappings in partial metric spaces
}

\author{
Laila A. Alnaser ${ }^{\mathrm{a}}$, Durdana Lateef ${ }^{\mathrm{a}}$, Jamshaid Ahmad ${ }^{\mathrm{b}, *}$ \\ ${ }^{a}$ Deanery of Academic Services, Taibah University, Al-Madinah Al-Munawwarah, 41411, Kingdom of Saudi Arabia. \\ ${ }^{b}$ Department of Mathematics, University of Jeddah, P. O. Box 80327, Jeddah 21589, Kingdom of Saudi Arabia.
}

\begin{abstract}
The aim of this paper is to prove common fixed point theorems for compatible mappings of type (A) for three self mappings satisfying certain contractive conditions and its topological properties in partial metric spaces.
\end{abstract}

Keywords: Fixed point, self mappings, compatibility of type (A), partial metric space.

2010 MSC: 47H10, 54H25.

(C)2018 All rights reserved.

\section{Introduction and preliminaries}

Matthews [7] introduced the notion of partial metric space and extended the Banach contraction principle to the class of complete partial metric space. After remarkable contribution of Matthews, many authors have studied partial metric space and its topological properties. Matthews discussed not only the general topological properties of partial metric spaces, but also some properties of convergence of sequences. The concept of compatible mapping was introduced by Jungck in the year 1986 [4] and proved that weakly commuting mappings are compatible mappings. In 1993, Jungck et al. [5] introduced compatible of type (A) and proved common fixed point. In this paper we obtain common fixed points of contractive type self mappings on partial metric spaces which cannot be deduced from the corresponding results in metric spaces. An example is also established to show that our result is a real generalizations of analogous results for metric spaces.

We begin with some basic known definitions and results which will be used in the sequel. Throughout this article, $\mathbb{N}, \mathbb{R}^{+}$, and $\mathbb{R}$ denote the set of natural numbers, the set of positive real numbers, and the set of real numbers, respectively.

\footnotetext{
*Corresponding author

Email addresses: alnaser_layla@yahoo.com (Laila A. Alnaser), drdurdanamaths@gmail.com (Durdana Lateef),

jamshaid_jasim@yahoo.com (Jamshaid Ahmad)

doi: $10.22436 /$ jmcs.018.03.09
}

Received: 2018-03-27 Revised: 2018-05-12 Accepted: 2018-05-31 
Definition 1.1. A pair $(S, T)$ of self mappings of a metric space $(X, d)$ is said to be compatible mappings, if

$$
\lim _{n \rightarrow \infty} d\left(S T x_{n}, T S x_{n}\right)=0
$$

whenever $\left\{x_{n}\right\}$ is a sequence in $X$ such that

$$
\lim _{n \rightarrow \infty} S x_{n}=\lim _{n \rightarrow \infty} T x_{n}=t
$$

for some $t \in X$.

In 1993, Jungck et al. [5] introduced the notion of compatible mappings of type (A) in such a way.

Definition 1.2. A pair $(S, T)$ of self mappings of a metric space $(X, d)$ is said to be compatible mappings of type (A), if

$$
\lim _{n \rightarrow \infty} d\left(S T x_{n}, T T x_{n}\right)=0 \text { and } \lim _{n \rightarrow \infty} d\left(T S x_{n}, T T x_{n}\right)=0
$$

whenever $\left\{x_{n}\right\}$ is a sequence in $X$ such that

$$
\lim _{n \rightarrow \infty} S x_{n}=\lim _{n \rightarrow \infty} T x_{n}=t
$$

for some $t \in X$.

Matthews [7] gave the concept of partial metric space in this way.

Definition 1.3. A partial metric on a nonempty set $X$ is a function $p: X \times X \rightarrow[0, \infty)$ such that for all $x, y, z \in X:$

$\left(P_{1}\right) p(x, x)=p(y, y)=p(x, y)$ if and only if $x=y$,

$\left(P_{2}\right) p(x, x) \leqslant p(x, y)$,

$\left(P_{3}\right) p(x, y)=p(y, x)$

$\left(P_{4}\right) p(x, z) \leqslant p(x, y)+p(y, z)-p(y, y)$.

The pair $(X, p)$ is then called a partial metric space. Also, each partial metric $p$ on $X$ generates a $T_{0}$ topology $\tau_{p}$ on $X$ with a base of the family of open $p$-balls $\left\{B_{p}(x, r): x \in X, r>0\right\}$, where $B_{p}(x, r)=\{y \in$ $X: p(x, y)<p(x, x)+r\}$. If $(X, p)$ is a partial metric space, then the function $p^{s}: X \times X \rightarrow \mathbb{R}^{+}$given by $p^{s}(x, y)=2 p(x, y)-p(x, x)-p(y, y), x, y \in X$, is a metric on $X$. A basic example of a partial metric space is the pair $\left(R^{+}, p\right)$, where $p(x, y)=\max \{x, y\}$ for all $x, y \in R^{+}$.

Lemma 1.4 ([7]). Let (X, $\mathrm{p})$ be a partial metric space, then we have the following.

1. A sequence $\left\{x_{n}\right\}$ in a partial metric space $(X, p)$ converges to a point $x \in X$ if and only if $\lim _{n \rightarrow \infty} p\left(x, x_{n}\right)=$ $\mathrm{p}(\mathrm{x}, \mathrm{x})$.

2. A sequence $\left\{x_{n}\right\}$ in a partial metric space $(X, p)$ is called a Cauchy sequence if the $\lim _{n, m \rightarrow \infty} p\left(x_{n}, x_{m}\right)$ exists and is finite.

3. A partial metric space $(X, p)$ is said to be complete if every Cauchy sequence $\left\{x_{n}\right\}$ in $X$ converges to a point $x \in X$, that is $p(x, x)=\lim _{n \rightarrow \infty} p\left(x_{n}, x_{m}\right)$.

4. A partial metric space $(X, p)$ is complete if and only if the metric space $\left(X, p^{s}\right)$ is complete. Furthermore, $\lim _{n \rightarrow \infty} p^{s}\left(x_{n}, z\right)=0$ if and only if $p(z, z)=\lim _{n \rightarrow \infty} p\left(x_{n}, z\right)=\lim _{n, m \rightarrow \infty} p\left(x_{n}, x_{m}\right)$. 


\section{Main results}

Now we state our main result.

Theorem 2.1. Let $(X, p)$ be a complete partial metric space and $S, T, f: X \rightarrow X$ be self-mappings satisfying the following assertions:

(i) $S(X) \cup T(X) \subseteq f(X)$;

(ii) there exist continuous mappings $\mathrm{a}, \mathrm{b}: \mathrm{X} \rightarrow[0,1)$ such that for all $\mathrm{x}, \mathrm{y} \in \mathrm{X}$,

(a) $a(S x) \leqslant a(f x)$ and $b(S x) \leqslant b(f x)$;

(b) $a(T x) \leqslant a(f x)$ and $b(T x) \leqslant b(f x)$;

(c) $a(f x)+2 b(f x)<1$;

(d)

$$
p(S x, T y) \leqslant a(f x) \max \{p(f x, f y), p(f x, S x), p(f y, T y)\}+b(f x)\{p(f x, T y)+p(f y, S x)\} ;
$$

(iii) one of the pairs $(S, f)$ or $(T, f)$ is compatible of type $(A)$;

(iv) the mapping $f$ is continuous.

Then the mappings $\mathrm{S}, \mathrm{T}$, and $\mathrm{f}$ have a unique common fixed point.

Proof. Let $x_{0}$ be an arbitrary point of $X$. We define the sequence $\left\{f x_{n}\right\}$ by

$$
f x_{2 n+1}=S x_{2 n} \text { and } f x_{2 n+2}=T x_{2 n+1}
$$

for all $n=0,1,2, \ldots$. From the inequality (2.1), we have

$$
\begin{aligned}
p\left(f x_{2 n+1}, f x_{2 n+2}\right)= & p\left(S x_{2 n}, T x_{2 n+1}\right) \\
\leqslant & a\left(f x_{2 n}\right) \max \left\{p\left(f x_{2 n}, f x_{2 n+1}\right), p\left(f x_{2 n}, S x_{2 n}\right), p\left(f x_{2 n+1}, T x_{2 n+1}\right)\right\} \\
& +b\left(f x_{2 n}\right)\left\{p\left(f x_{2 n}, T x_{2 n+1}\right)+p\left(f x_{2 n+1}, S x_{2 n}\right)\right\} \\
= & a\left(T x_{2 n-1}\right) \max \left\{p\left(f x_{2 n}, f x_{2 n+1}\right), p\left(f x_{2 n}, f x_{2 n+1}\right), p\left(f x_{2 n+1}, f x_{2 n+2}\right)\right\} \\
& +b\left(T x_{2 n-1}\right)\left\{p\left(f x_{2 n}, f x_{2 n+2}\right)+p\left(f x_{2 n+1}, f x_{2 n+1}\right)\right\} \\
\leqslant & a\left(f x_{2 n-1}\right) \max \left\{p\left(f x_{2 n}, f x_{2 n+1}\right), p\left(f x_{2 n}, f x_{2 n+1}\right), p\left(f x_{2 n+1}, f x_{2 n+2}\right)\right\} \\
& +b\left(f x_{2 n-1}\right)\left\{p\left(f x_{2 n}, f x_{2 n+2}\right)+p\left(f x_{2 n+1}, f x_{2 n+1}\right)\right\} \\
= & a\left(S x_{2 n-2}\right) \max \left\{p\left(f x_{2 n}, f x_{2 n+1}\right), p\left(f x_{2 n}, f x_{2 n+1}\right), p\left(f x_{2 n+1}, f x_{2 n+2}\right)\right\} \\
& +b\left(S x_{2 n-2}\right)\left\{p\left(f x_{2 n}, f x_{2 n+2}\right)+p\left(f x_{2 n+1}, f x_{2 n+1}\right)\right\} \\
\leqslant & a\left(f x_{2 n-2}\right) \max \left\{p\left(f x_{2 n}, f x_{2 n+1}\right), p\left(f x_{2 n}, f x_{2 n+1}\right), p\left(f x_{2 n+1}, f x_{2 n+2}\right)\right\} \\
& +b\left(f x_{2 n-2}\right)\left\{p\left(f x_{2 n}, f x_{2 n+2}\right)+p\left(f x_{2 n+1}, f x_{2 n+1}\right)\right\} \\
\vdots & \\
\leqslant & a\left(f x_{0}\right) \max \left\{p\left(f x_{2 n}, f x_{2 n+1}\right), p\left(f x_{2 n}, f x_{2 n+1}\right), p\left(f x_{2 n+1}, f x_{2 n+2}\right)\right\} \\
& +b\left(f x_{0}\right)\left\{p\left(f x_{2 n}, f x_{2 n+2}\right)+p\left(f x_{2 n+1}, f x_{2 n+1}\right)\right\} .
\end{aligned}
$$

By using (P4), we get

$$
\begin{aligned}
p\left(f x_{2 n+1}, f x_{2 n+2}\right) \leqslant & a\left(f x_{0}\right) \max \left\{p\left(f x_{2 n}, f x_{2 n+1}\right), p\left(f x_{2 n+1}, f x_{2 n+2}\right)\right\} \\
& +b\left(f x_{0}\right)\left\{p\left(f x_{2 n}, f x_{2 n+1}\right)+p\left(f x_{2 n+1}, f x_{2 n+2}\right)\right\}-p\left(f x_{2 n+1}, f x_{2 n+1}\right),
\end{aligned}
$$

which can be written as

$$
\begin{aligned}
p\left(f x_{2 n+1}, f x_{2 n+2}\right) \leqslant & a\left(f x_{0}\right) \max \left\{p\left(f x_{2 n}, f x_{2 n+1}\right), p\left(f x_{2 n+1}, f x_{2 n+2}\right)\right\} \\
& +b\left(f x_{0}\right)\left\{p\left(f x_{2 n}, f x_{2 n+1}\right)+p\left(f x_{2 n+1}, f x_{2 n+2}\right)\right\} .
\end{aligned}
$$

Now two cases arise: 
Case i. If $\max \left\{p\left(f x_{2 n}, f x_{2 n+1}\right), p\left(f x_{2 n+1}, f x_{2 n+2}\right)\right\}=p\left(f x_{2 n}, f x_{2 n+1}\right)$, then from previous inequality, we get $p\left(f x_{2 n+1}, f x_{2 n+2}\right) \leqslant a\left(f x_{0}\right) p\left(f x_{2 n}, f x_{2 n+1}\right)+b\left(f x_{0}\right)\left\{p\left(f x_{2 n}, f x_{2 n+1}\right)+p\left(f x_{2 n+1}, f x_{2 n+2}\right)\right\}$,

which can be written in simplifying form as

$$
p\left(f x_{2 n+1}, f x_{2 n+2}\right) \leqslant\left(\frac{a\left(f x_{0}\right)+b\left(f x_{0}\right)}{1-b\left(f x_{0}\right)}\right) p\left(f x_{2 n}, f x_{2 n+1}\right) .
$$

Let $\left(\frac{a\left(f x_{0}\right)+b\left(f x_{0}\right)}{1-b\left(f x_{0}\right)}\right)=\lambda_{1}$, then from above we have

$$
p\left(f x_{2 n+1}, f x_{2 n+2}\right) \leqslant \lambda_{1} p\left(f x_{2 n}, f x_{2 n+1}\right) .
$$

Case ii. If $\max \left\{p\left(f x_{2 n}, f x_{2 n+1}\right), p\left(f x_{2 n+1}, f x_{2 n+2}\right)\right\}=p\left(f x_{2 n+1}, f x_{2 n+2}\right)$. Then from previous inequality, we get

$$
p\left(f x_{2 n+1}, f x_{2 n+2}\right) \leqslant a\left(f x_{0}\right) p\left(f x_{2 n+1}, f x_{2 n+2}\right)+b\left(f x_{0}\right)\left\{p\left(f x_{2 n}, f x_{2 n+1}\right)+p\left(f x_{2 n+1}, f x_{2 n+2}\right)\right\},
$$

which can be written in simplifying form as

$$
p\left(f x_{2 n+1}, f x_{2 n+2}\right) \leqslant\left(\frac{b\left(f x_{0}\right)}{1-a\left(f x_{0}\right)-b\left(f x_{0}\right)}\right) p\left(f x_{2 n}, f x_{2 n+1}\right) .
$$

Let $\left(\frac{b\left(f x_{0}\right)}{1-a\left(f x_{0}\right)-b\left(f x_{0}\right)}\right)=\lambda_{2}$, then from above we have

$$
p\left(f x_{2 n+1}, f x_{2 n+2}\right) \leqslant \lambda_{2} p\left(f x_{2 n}, f x_{2 n+1}\right) .
$$

As $a(x)+2 b(x)<1$, so we will have both $\lambda_{1}$ and $\lambda_{2}$ less than 1 . If we take $\lambda=\max \left\{\lambda_{1}, \lambda_{2}\right\}$, then from both cases we have

$$
p\left(f x_{2 n+1}, f x_{2 n+2}\right) \leqslant \lambda p\left(f x_{2 n}, f x_{2 n+1}\right) .
$$

If we repeat the same procedure as above, we get

$$
p\left(f x_{2 n}, f x_{2 n+1}\right) \leqslant \lambda p\left(f x_{2 n-1}, f x_{2 n}\right) .
$$

Inductively, we have

$$
p\left(f x_{2 n+1}, f x_{2 n+2}\right) \leqslant \lambda^{2 n+1} p\left(f x_{0}, f x_{1}\right) .
$$

Hence

$$
p\left(f x_{n}, f x_{n+1}\right) \leqslant \lambda^{n} p\left(f x_{0}, f x_{1}\right) .
$$

Now we prove that the sequence $\left\{f x_{n}\right\}$ is a Cauchy sequence in $(X, p)$.

Let $m, n \in \mathbb{N}$, with $m>n$ and consider

$$
\begin{aligned}
p\left(f x_{n}, f x_{n+m}\right) \leqslant & p\left(f x_{n}, f x_{n+1}\right)+p\left(f x_{n+1}, f x_{n+2}\right)+\cdots+p\left(f x_{n+m-1}, f x_{n+m}\right) \\
& -\left(p\left(f x_{n+1}, f x_{n+1}\right)+p\left(f x_{n+2}, f x_{n+2}\right)+\cdots+p\left(f x_{n+m-1}, f x_{n+m-1}\right)\right) .
\end{aligned}
$$

By using the inequality (2.2), we get

$$
p\left(f x_{n}, f x_{n+m}\right) \leqslant\left[\lambda^{n}+\lambda^{n+1}+\cdots+\lambda^{n+m-1}\right] p\left(f x_{0}, f x_{1}\right) \leqslant \frac{\lambda^{n}}{1-\lambda} p\left(f x_{0}, f x_{1}\right) .
$$

As $\lambda<1$, so we have $p\left(f x_{n}, f x_{n+m}\right) \rightarrow 0$ as $m, n \rightarrow \infty$. Hence

$$
\lim _{n, m \rightarrow \infty} p\left(f x_{n}, f x_{n+m}\right)=0 .
$$


As we have the following relation

$$
p^{s}(x, y)=2 p(x, y)-p(x, x)-p(y, y) .
$$

So we can write it as

$$
p^{s}\left(f x_{n}, f x_{n+m}\right)=2\left(f x_{n}, f x_{n+m}\right)-\left(f x_{n}, f x_{n}\right)-\left(f x_{n+m}, f x_{n+m}\right) \leqslant 2\left(f x_{n}, f x_{n+m}\right) .
$$

Applying (2.3), we have

$$
\lim _{n, m \rightarrow \infty} p^{s}\left(f x_{n}, f x_{n+m}\right)=0
$$

Hence the sequence $\left\{f x_{n}\right\}$ is a Cauchy sequence in $\left(X, p^{s}\right)$. Since $(X, p)$ is complete, so the corresponding metric space $\left(X, p^{s}\right)$ is also complete. Therefore, the sequence $\left\{f x_{n}\right\}$ converges to some $z \in X$ with respect to the metric $p^{s}$ that is

$$
\lim _{n \rightarrow \infty} p^{s}\left(f x_{n}, z\right)=0
$$

Since

Therefore

$$
p\left(f x_{n}, f x_{n}\right) \leqslant p\left(f x_{n}, f x_{n+1}\right) \leqslant \lambda^{n} p\left(f x_{0}, f x_{1}\right) \rightarrow 0 \text { as } n \rightarrow \infty .
$$

$$
p(z, z) \leqslant \lim _{n \rightarrow \infty} p\left(f x_{n}, z\right) \leqslant \lim _{n, m \rightarrow \infty} p\left(f x_{n}, f x_{n+m}\right)=0 .
$$

Thus the sequence $\left\{f x_{n}\right\}$ is a Cauchy sequence in $(X, p)$. Since $(X, p)$ is complete, so there exists some $z \in X$ such that $\lim _{n \rightarrow \infty} f x_{n}=z$. It follows that the sequences $\left\{S x_{n}\right\}$ and $\left\{T x_{n+1}\right\}$ also converge to $z$. First we suppose that the pair $(f, S)$ is compatible of type $(A)$. Then from the inequality (2.1), we have

$$
\begin{aligned}
p\left(S f x_{2 n}, T x_{2 n+1}\right) \leqslant & a\left(f f x_{2 n}\right) \max \left\{p\left(f f x_{2 n}, f x_{2 n+1}\right), p\left(f f x_{2 n}, S f x_{2 n}\right), p\left(f x_{2 n+1}, T x_{2 n+1}\right)\right\} \\
& +b\left(f f x_{2 n}\right)\left\{p\left(f f x_{2 n}, T x_{2 n+1}\right)+p\left(f x_{2 n+1}, S f x_{2 n}\right)\right\} .
\end{aligned}
$$

Since the mapping $f$ is continuous, so we have $f f x_{2 n} \rightarrow f z$ and $f S x_{2 n} \rightarrow f z$ as $n \rightarrow \infty$. From the supposition that the pair $(f, S)$ is compatible of type $(A)$, we have

$$
f S x_{2 n} \rightarrow f z, S f x_{2 n} \rightarrow f z, \text { and } f f x_{2 n} \rightarrow f z
$$

as $n \rightarrow \infty$. So letting $n \rightarrow \infty$ in above inequality, we have

$$
p(f z, z) \leqslant a(f z) \max \{p(f z, z), p(f z, f z), p(z, z)\}+b(f z)\{p(f z, z)+p(z, f z)\} .
$$

As $p(x, x) \leqslant p(x, y)$, so we can write

$$
p(f z, z) \leqslant a(f z) \max \{p(f z, z), p(f z, z), p(f z, z)\}+b(f z)\{p(f z, z)+p(z, f z)\} .
$$

Thus after simplifying, we have

$$
(1-a(f z)-2 b(f z)) p(f z, z) \leqslant 0
$$

It follows that $f z=z$.

Now from the inequality (2.1), we have

$$
p\left(S z, T x_{2 n+1}\right) \leqslant a(z) \max \left\{p\left(f z, f x_{2 n+1}\right), p(f z, S z), p\left(f x_{2 n+1}, T x_{2 n+1}\right)\right\}+b(z)\left\{p\left(f z, T x_{2 n+1}\right)+p\left(f x_{2 n+1}, S z\right)\right\} .
$$

Letting $n \rightarrow \infty$, we have

$$
\mathrm{p}(\mathrm{S} z, z) \leqslant \mathrm{a}(z) \max \{\mathrm{p}(\mathrm{f} z, z), \mathrm{p}(\mathrm{f} z, \mathrm{~S} z), \mathrm{p}(z, z)\}+\mathrm{b}(z)\{\mathrm{p}(\mathrm{f} z, z)+\mathrm{p}(z, \mathrm{~S} z)\} .
$$

As $f z=z$, so one can easily get $S z=z$ from above. Similarly, we consider

$$
p\left(S x_{2 n}, T z\right) \leqslant a(z) \max \left\{p\left(f x_{2 n}, f z\right), p\left(f x_{2 n}, S x_{2 n}\right), p(f z, T z)\right\}+b(z)\left\{p\left(f x_{2 n}, T z\right)+p\left(f z, S x_{2 n}\right)\right\} .
$$


Letting $n \rightarrow \infty$, we have

$$
p(z, T z) \leqslant a(z) \max \{p(z, f z), p(z, z), p(f z, T z)\}+b(z)\{p(z, T z)+p(f z, z)\} .
$$

Using the fact as $f z=z$, we can obtain $T z=z$. Thus we have $S z=T z=f z=z$ that is $z$ is a common fixed point of $S, T$, and $f$. Similarly we can prove that $z$ is a common fixed point of $S, T$, and $f$ when the pair $(f, T)$ is compatible of type $(A)$.

Now we prove the uniqueness of this theorem. Let $w$ be another common fixed point of $S, T$, and $f$ other than $z$. Then $S w=T w=f w=w$ and $S z=T z=f z=z$ but $w \neq z$. Now from the inequality (2.1), we have

$$
\begin{aligned}
\mathrm{p}(z, w)=\mathrm{p}(\mathrm{S} z, \mathrm{~T} w) & \leqslant \mathrm{a}(z) \max \{\mathrm{p}(\mathrm{f} z, \mathrm{f} w), \mathrm{p}(\mathrm{f} z, \mathrm{~S} z), \mathrm{p}(\mathrm{f} w, \mathrm{~T} w)\}+\mathrm{b}(z)\{\mathrm{p}(\mathrm{f} z, \mathrm{~T} w)+\mathrm{p}(\mathrm{f} w, \mathrm{~S} z)\} \\
& =\mathrm{a}(z) \max \{\mathrm{p}(z, w), \mathrm{p}(z, z), \mathrm{p}(w, w)\}+\mathrm{b}(z)\{\mathrm{p}(z, w)+\mathrm{p}(w, z)\} \\
& =\mathrm{a}(z) \mathrm{p}(z, w)+2 \mathrm{~b}(z) \mathrm{p}(z, w)=(\mathrm{a}(z)+2 \mathrm{~b}(z)) \mathrm{p}(z, w)
\end{aligned}
$$

which is a contradiction to the fact that $z \neq w$. Thus $z=w$. This completes the proof.

Corollary 2.2. Let $(X, p)$ be a complete partial metric space and $S, f: X \rightarrow X$ be self-mappings satisfying the following assertions:

(i) $\mathrm{S}(\mathrm{X}) \subseteq \mathrm{f}(\mathrm{X})$;

(ii) there exist continuous mappings $\mathrm{a}, \mathrm{b}: \mathrm{X} \rightarrow[0,1)$ such that for all $\mathrm{x}, \mathrm{y} \in \mathrm{X}$,

(a) $a(S x) \leqslant a(f x)$ and $b(S x) \leqslant b(f x)$;

(b) $a(f x)+2 b(f x)<1$;

(c) $p(S x, S y) \leqslant a(f x) \max \{p(f x, f y), p(f x, S x), p(f y, S y)\}+b(f x)\{p(f x, S y)+p(f y, S x)\} ;$

(iii) the pair $(\mathrm{S}, \mathrm{f})$ is compatible of type $(\mathrm{A})$;

(iv) the mapping $f$ is continuous.

Then the mappings $\mathrm{S}$ and $\mathrm{f}$ have a unique common fixed point.

Corollary 2.3. Let $(X, p)$ be a complete partial metric space and $\mathrm{S}, \mathrm{T}, \mathrm{f}: \mathrm{X} \rightarrow \mathrm{X}$ be self-mappings satisfying the following assertions:

(i) $S(X) \cup T(X) \subseteq f(X)$;

(ii) there exist constants $a, b \in[0,1)$ such that for all $x, y \in X$,

$$
p(S x, T y) \leqslant a \max \{p(f x, f y), p(f x, S x), p(f y, T y)\}+b\{p(f x, T y)+p(f y, S x)\}
$$

with $a+2 b<1$;

(iii) one of the pairs $(\mathrm{S}, \mathrm{f})$ or $(\mathrm{T}, \mathrm{f})$ is compatible of type $(\mathrm{A})$;

(iv) the mapping $f$ is continuous.

Then the mappings $\mathrm{S}, \mathrm{T}$, and $\mathrm{f}$ have a unique common fixed point.

Corollary 2.4. Let $(X, p)$ be a complete partial metric space and $\mathrm{S}, \mathrm{f}: \mathrm{X} \rightarrow \mathrm{X}$ be self-mappings satisfying the following assertions:

(i) $S(X) \subseteq f(X)$;

(ii) there exist constants $a, b \in[0,1)$ such that for all $x, y \in X$,

$$
p(S x, S y) \leqslant a \max \{p(f x, f y), p(f x, S x), p(f y, S y)\}+b\{p(f x, S y)+p(f y, S x)\}
$$

with $a+2 b<1$;

(iii) the pair $(\mathrm{S}, \mathrm{f})$ is compatible of type (A);

(iv) the mapping $f$ is continuous. 
Then the mappings $\mathrm{S}$ and $\mathrm{f}$ have a unique common fixed point.

Remark 2.5. By taking different mappings as Identity mapping in our main result we can get variety of corollaries.

Theorem 2.6. Let $(X, p)$ be a complete partial metric space and $S, T, f: X \rightarrow X$ be self-mappings satisfying the following assertions:

(i) $S(X) \cup T(X) \subseteq f(X)$;

(ii) there exist continuous mappings $\mathrm{b}: \mathrm{X} \rightarrow[0,1)$ such that for all $\mathrm{x}, \mathrm{y} \in \mathrm{X}$,

(a) $b(S x) \leqslant b(f x)$;

(b) $b(T x) \leqslant b(f x)$;

(c) $b(f x)<\frac{1}{2}$

(d) $p(S x, T y)^{\prime} \leqslant b(f x)\{p(f x, T y)+p(f y, S x)\}$

(iii) one of the pairs $(\mathrm{S}, \mathrm{f})$ or $(\mathrm{T}, \mathrm{f})$ is compatible of type $(\mathrm{A})$;

(iv) the mapping $f$ is continuous.

Then the mappings $\mathrm{S}, \mathrm{T}$, and f have a unique common fixed point.

Theorem 2.7. Let $(\mathrm{X}, \mathrm{p})$ be a complete partial metric space and $\mathrm{S}, \mathrm{T}, \mathrm{f}: \mathrm{X} \rightarrow \mathrm{X}$ be self-mappings satisfying the following assertions:

(i) $S(X) \cup T(X) \subseteq f(X)$;

(ii) $\mathrm{p}(\mathrm{Sx}, \mathrm{Ty}) \leqslant \mathrm{b}\{\mathrm{p}(\mathrm{f} x, \mathrm{Ty})+\mathrm{p}(\mathrm{fy}, \mathrm{Sx})\}$ for all $\mathrm{x}, \mathrm{y} \in \mathrm{X}$ and $0 \leqslant \mathrm{~b}<\frac{1}{2}$;

(iii) one of the pairs $(\mathrm{S}, \mathrm{f})$ or $(\mathrm{T}, \mathrm{f})$ is compatible of type $(\mathrm{A})$;

(iv) the mapping $f$ is continuous.

Then the mappings $\mathrm{S}, \mathrm{T}$, and $\mathrm{f}$ have a unique common fixed point.

\section{Common fixed point results for F-contraction}

F-contraction is recent development in the field of fixed point theory and has lot of generalizations in current research. This concept was given by Wardowski [9] in 2012. We begin this section with the following basic definition of F-contraction.

Definition 3.1. Let $\mathrm{F}: \mathbb{R}^{+} \rightarrow \mathbb{R}$ be a mapping satisfying the following conditions:

$\left(F_{1}\right) F$ is strictly increasing;

( $\left.F_{2}\right)$ for all sequence $\left\{\alpha_{n}\right\} \subseteq R^{+}, \lim _{n \rightarrow \infty} \alpha_{n}=0$ if and only if $\lim _{n \rightarrow \infty} F\left(\alpha_{n}\right)=-\infty$;

$\left(\mathrm{F}_{3}\right)$ there exists $0<\mathrm{k}<1$ such that $\lim _{n \rightarrow 0^{+}} \alpha^{k} \mathrm{~F}(\alpha)=0$.

A mapping $T: X \rightarrow X$ is said to be an F-contraction if there exists $\tau \in \mathbb{R}^{+}$such that for all $x, y \in X$;

$$
d(T x, T y)>0 \Longrightarrow \tau+F(d(T x, T y)) \leqslant F(d(x, y))
$$

To be consistent with Wardowski [9], we denote by $\digamma$ the set of all functions $F: \mathbb{R}^{+} \rightarrow \mathbb{R}$ satisfying the above conditions. Later on many authors generalized this result in a different way in various generalized metric spaces. For more details in this direction we refer the reader to $[1,3,2,6,8,10]$.

In this paper, we establish a common fixed point theorem for three self mappings in the setting of complete partial metric spaces and obtained different results as corollaries of our main result. We also give a remark with a suitable example that our result can not be derived from the ordinary metric space.

Now we state and prove our main result for three self mappings in partial metric space.

Theorem 3.2. Let $(X, p)$ be a complete partial metric space and $\mathrm{S}, \mathrm{T}, \mathrm{f}: \mathrm{X} \rightarrow \mathrm{X}$ be self-mappings satisfying the following assertions:

(i) $S(X) \cup T(X) \subseteq f(X)$;

(ii) $p(S x, T y)>0$ implies

for all $x, y \in X$ and $F \in \digamma$;

$$
\tau+F(p(S x, T y)) \leqslant F(p(f x, f y))
$$


(iii) one of the pairs $(\mathrm{S}, \mathrm{f})$ or $(\mathrm{T}, \mathrm{f})$ is compatible of type (A);

(iv) the mapping $f$ is continuous.

Then $\mathrm{S}, \mathrm{T}$, and $\mathrm{f}$ have a unique common fixed point.

Proof. Let $x_{0}$ be an arbitrary point of $X$. As we done in previous theorem, we define the sequence $\left\{f x_{n}\right\}$ by

$$
f x_{2 n+1}=S x_{2 n} \text { and } f x_{2 n+2}=T x_{2 n+1}
$$

for all $n=0,1,2, \ldots$.From the inequality (3.1), we have

$$
\tau+F\left(p\left(f x_{2 n+1}, f x_{2 n+2}\right)\right)=\tau+F\left(p\left(S x_{2 n}, T x_{2 n+1}\right)\right) \leqslant F\left(p\left(f x_{2 n}, f x_{2 n+1}\right)\right),
$$

which implies that

$$
F\left(p\left(f x_{2 n+1}, f x_{2 n+2}\right)\right) \leqslant F\left(p\left(f x_{2 n}, f x_{2 n+1}\right)\right)-\tau .
$$

Similarly from (3.1), we have

$$
\begin{aligned}
\tau+F\left(p\left(f x_{2 n+2}, f x_{2 n+3}\right)\right) & =\tau+F\left(p\left(T x_{2 n+1}, S x_{2 n+2}\right)\right) \\
& =\tau+F\left(p\left(S x_{2 n+2}, T x_{2 n+1}\right)\right) \leqslant F\left(p\left(f x_{2 n+2}, f x_{2 n+1}\right)\right)=F\left(p\left(f x_{2 n+1}, f x_{2 n+2}\right)\right),
\end{aligned}
$$

which implies that

$$
F\left(p\left(f x_{2 n+2}, f x_{2 n+3}\right)\right) \leqslant F\left(p\left(f x_{2 n+1}, f x_{2 n+2}\right)\right)-\tau .
$$

Thus for all $n=1,2, \ldots$ we have

$$
F\left(p\left(f x_{n}, f x_{n+1}\right)\right) \leqslant F\left(p\left(f x_{n-1}, f x_{n}\right)\right)-\tau \leqslant F\left(p\left(f x_{n-2}, f x_{n-1}\right)\right)-2 \tau \leqslant \cdots \leqslant F\left(p\left(f x_{0}, f x_{1}\right)\right)-n \tau
$$

for all $n \in \mathbb{N}$. Since $F \in \digamma$, so by taking limit as $n \rightarrow \infty$ in (3.2) we have,

$$
\lim _{n \rightarrow \infty} F\left(p\left(f x_{n}, f x_{n+1}\right)\right)=-\infty \Longleftrightarrow \lim _{n \rightarrow \infty} p\left(f x_{n}, f x_{n+1}\right)=0
$$

Now from (F3), there exists $0<k<1$ such that,

$$
\lim _{n \rightarrow \infty}\left[p\left(f x_{n}, f x_{n+1}\right)\right]^{k} F\left(p\left(f x_{n}, f x_{n+1}\right)\right)=0 .
$$

By (3.4), we have

$$
\begin{aligned}
& p\left(f x_{n}, f x_{n+1}\right)^{k} F\left(p\left(f x_{n}, f x_{n+1}\right)\right)-p\left(f x_{n}, f x_{n+1}\right)^{k} F\left(p\left(f x_{0}, f x_{1}\right)\right) \\
& \left.\quad<p\left(f x_{n}, f x_{n+1}\right)^{k}\left[F\left(p\left(f x_{0}, f x_{1}\right)-n \tau\right)\right)-F\left(p\left(f x_{0}, f x_{1}\right)\right)\right]=-n \tau\left[p\left(f x_{n}, f x_{n+1}\right)\right]^{k} \leqslant 0 .
\end{aligned}
$$

By taking limit as $n \rightarrow \infty$ in (3.5) and applying (3.3) and (3.4), we have,

$$
\lim _{n \rightarrow \infty} n\left[p\left(f x_{n}, f x_{n+1}\right)\right]^{k}=0 \text {. }
$$

It follows from (3.6) that there exists $n_{1} \in \mathbb{N}$ such that,

$$
n\left[p\left(f x_{n}, f x_{n+1}\right)\right]^{k} \leqslant 1
$$

for all $n>n_{1}$. This implies,

$$
p\left(f x_{n}, f x_{n+1}\right) \leqslant \frac{1}{n^{1 / k}}
$$

for all $n>n_{1}$. Now we prove that $\left\{x_{n}\right\}$ is a Cauchy sequence. For $m>n>n_{1}$ we have,

$$
p\left(f x_{n}, f x_{n+m}\right) \leqslant \sum_{i=n}^{n+m-1} p\left(f x_{i}, f x_{i+1}\right) \leqslant \sum_{i=n}^{n+m-1} \frac{1}{i^{1 / k}} .
$$


Since, $0<k<1$, then $\sum_{i=1}^{\infty} \frac{1}{i^{1 / k}}$ converges, so we have $p\left(f x_{n}, f x_{n+m}\right) \rightarrow 0$ as $m, n \rightarrow \infty$. Hence

$$
\lim _{n, m \rightarrow \infty} p\left(f x_{n}, f x_{n+m}\right)=0 .
$$

As we have the following relation

$$
p^{s}(x, y)=2 p(x, y)-p(x, x)-p(y, y),
$$

so we can write it as

$$
p^{s}\left(f x_{n}, f x_{n+m}\right)=2\left(f x_{n}, f x_{n+m}\right)-\left(f x_{n}, f x_{n}\right)-\left(f x_{n+m}, f x_{n+m}\right) \leqslant 2\left(f x_{n}, f x_{n+m}\right) .
$$

Applying (3.7), we have

$$
\lim _{n, m \rightarrow \infty} p^{s}\left(f x_{n}, f x_{n+m}\right)=0
$$

Hence the sequence $\left\{f x_{n}\right\}$ is a Cauchy sequence in $\left(X, p^{s}\right)$. Since $(X, p)$ is complete, so the corresponding metric space $\left(X, p^{s}\right)$ is also complete. Therefore, the sequence $\left\{f x_{n}\right\}$ converges to some $z \in X$ with respect to the metric $p^{s}$ that is

$$
\lim _{n \rightarrow \infty} p^{s}\left(f x_{n}, z\right)=0
$$

Since

$$
p\left(f x_{n}, f x_{n}\right) \leqslant p\left(f x_{n}, f x_{n+1}\right) \leqslant \frac{1}{n^{1 / k}} \rightarrow 0 \text { as } n \rightarrow \infty .
$$

Therefore

$$
p(z, z) \leqslant \lim _{n \rightarrow \infty} p\left(f x_{n}, z\right) \leqslant \lim _{n, m \rightarrow \infty} p\left(f x_{n}, f x_{n+m}\right)=0 .
$$

Thus the sequence $\left\{f x_{n}\right\}$ is a Cauchy sequence in $(X, p)$. Since $(X, p)$ is complete, so there exists some $z \in X$ such that $\lim _{n \rightarrow \infty} f x_{n}=z$. It follows that the sequences $\left\{S x_{n}\right\}$ and $\left\{T x_{n+1}\right\}$ also converge to $z$. First we suppose that the pair $(f, S)$ is compatible of type $(A)$ and contrary suppose that $f z \neq z$. Then from the inequality (3.1), we have

$$
\tau+F\left(p\left(S f x_{2 n}, T x_{2 n+1}\right)\right) \leqslant F\left(p\left(f f x_{2 n}, f x_{2 n+1}\right)\right) .
$$

As $F$ is strictly increasing, so we get

$$
p\left(S f x_{2 n}, T x_{2 n+1}\right) \leqslant p\left(f f x_{2 n}, f x_{2 n+1}\right) .
$$

Since the mapping $f$ is continuous, so we have $f f x_{2 n} \rightarrow f z$ and $f S x_{2 n} \rightarrow f z$ as $n \rightarrow \infty$. From the supposition that the pair $(f, S)$ is compatible of type $(A)$, we have

$$
f S x_{2 n} \rightarrow f z, S f x_{2 n} \rightarrow f z \text { and } f f x_{2 n} \rightarrow f z
$$

as $n \rightarrow \infty$. So letting $n \rightarrow \infty$ in inequality (3.8), we have

$$
p(f z, z)<p(f z, z)
$$

which is a contradiction. Thus $f z=z$.

Now from the inequality (3.1), we have

$$
\tau+F\left(p\left(S z, T x_{2 n+1}\right)\right) \leqslant F\left(p\left(f z, f x_{2 n+1}\right)\right) .
$$

As F is strictly increasing, so we get

$$
p\left(S z, T x_{2 n+1}\right) \leqslant p\left(f z, f x_{2 n+1}\right) .
$$

Letting $\mathrm{n} \rightarrow \infty$, we have

$$
p(S z, z) \leqslant p(f z, z) .
$$


As $f z=z$, so one can easily get $S z=z$ from above. Similarly, we consider

$$
\tau+F\left(p\left(S x_{2 n}, T z\right)\right) \leqslant F\left(p\left(f x_{2 n}, f z\right)\right) .
$$

As $F$ is strictly increasing, so we get

$$
p\left(S x_{2 n}, T z\right) \leqslant p\left(f x_{2 n}, f z\right)
$$

Letting $n \rightarrow \infty$, we have

$$
p(z, T z) \leqslant p(z, f z)
$$

Using the fact as $f z=z$, we can obtain $T z=z$. Thus we have $S z=T z=f z=z$ that is $z$ is a common fixed point of $S, T$, and $f$. Similarly we can prove that $z$ is a common fixed point of $S, T$, and $f$ when the pair $(f, T)$ is compatible of type (A).

Now we prove the uniqueness of this theorem. Let $w$ be another common fixed point of $\mathrm{S}, \mathrm{T}$, and $\mathrm{f}$ other than $z$. Then $S w=T w=f w=w$ and $S z=T z=f z=z$ but $w \neq z$. Now from the inequality (3.1), we have

$$
\tau+\mathrm{F}(\mathrm{p}(z, w))=\tau+\mathrm{F}(\mathrm{p}(\mathrm{S} z, \mathrm{~T} w)) \leqslant \mathrm{F}(\mathrm{p}(\mathrm{f} z, \mathrm{f} w))=\mathrm{F}(\mathrm{p}(z, w)),
$$

which is a contradiction to the fact that $z \neq w$, because $\tau>0$. Thus $z=w$. This completes the proof.

Corollary 3.3. Let $(X, p)$ be a complete partial metric space and $S, T: X \rightarrow X$ be self-mappings satisfying the following assertion:

$$
p(S x, T y)>0 \text { implies } \tau+F(p(S x, T y)) \leqslant F(p(x, y))
$$

for all $\mathrm{x}, \mathrm{y} \in \mathrm{X}$ and $\mathrm{F} \in F$. Then $\mathrm{S}$ and $\mathrm{T}$ have a unique common fixed point.

Proof. Taking $f=I$ (identity mapping) we have the result.

Corollary 3.4. Let $(X, p)$ be a complete partial metric space and $S, T: X \rightarrow X$ be self-mappings satisfying the following assertion:

$$
p(S x, T y) \leqslant \lambda p(x, y)
$$

for all $x, y \in X$ where $0 \leqslant \lambda<1$. Then $S$ and $\mathrm{T}$ have a unique common fixed point.

Remark 3.5. The above theorem cannot be deduced from similar result of metric spaces. Actually the contractive condition (3.9) for a pair $S, T: X \rightarrow X$ of mappings on a metric space $(X, d)$ that is

$$
\mathrm{d}(S x, T y) \leqslant \lambda d(x, y) \text { for all } x, y \in X
$$

is not attainable. Because $S \neq T$ implies that $S v \neq T v$, for some $v \in X$, then

$$
\mathrm{d}(\mathrm{S} v, \mathrm{~T} v)>0=\lambda \mathrm{d}(v, v)
$$

and condition (3.9) is not satisfied for $x=y=v$. However the same condition in partial metric space is feasible to find common fixed point result for a pair of mappings. This fact can been seen again in the following example.

Example 3.6. Let $X=[0,1]$ and $p(x, y)=\max \{x, y\}$ and $S, T: X \rightarrow X$ be defined by

$$
\mathrm{Sx}=\frac{1}{8} \mathrm{x}, \mathrm{T} x=\frac{3}{8} \mathrm{x} .
$$

Then

$$
d(S x, T y)=\max \left\{\frac{1}{8} x, \frac{3}{8} x\right\} \quad \text { and } \quad d(S x, T y)=\frac{1}{8} \max \{x, 3 y\} \leqslant \frac{5}{11} \max \{x, y\} \leqslant \lambda p(x, y) .
$$

Therefore, for $\lambda=\frac{3}{10}$ all the conditions of Corollary 3.4 are satisfied to find common fixed point of $S$ and $T$. However, note that for any metric $d$ on $X$

$$
\mathrm{d}(\mathrm{S} 1, \mathrm{~T} 1)=\mathrm{d}\left(\frac{1}{8}, \frac{3}{8}\right)>\lambda \mathrm{d}(1,1)=0 \text { for any } \lambda \in[0,1) .
$$

Therefore common fixed points of $S$ and $T$ cannot be obtained from an corresponding metric fixed point theorem. 


\section{Acknowledgment}

This article was funded by the Deanship of Scientific Research (DSR), Taibah University, Al-Madinah Al-Munawwarah. Therefore, first author acknowledges with thanks DSR, TU for financial support.

\section{References}

[1] J. Ahmad, A. Al-Rawashdeh, A. Azam, New fixed point theorems for generalized F-contractions in complete metric spaces, Fixed Point Theory Appl., 2015 (2015), 18 pages. 3

[2] N. Hussain, J. Ahmad, A. Azam, On Suzuki-Wardowski type fixed point theorems, J. Nonlinear Sci. Appl., 8 (2015), 1095-1111. 3

[3] N. Hussain, J. Ahmad, L. Ćirić, A. Azam, Coincidence point theorems for generalized contractions with application to integral equations, Fixed Point Theory Appl., 2015 (2015), 13 pages. 3

[4] G. Jungck, Compatible mappings and common fixed points, Internat. J. Math. Math. Sci., 9 (1986), 771-779. 1

[5] G. Jungck, P. P. Murthy, Y. Cho, Compatible mappings of type (A) and common fixed points, Math. Japon., 38 (1993), 381-390. 1, 1

[6] S. U. Khan, M. Arshad, A. Hussain, M. Nazam, Two new types of fixed point theorems for F-contraction, J. Adv. Stud. Topol., 7 (2016), 251-260. 3

[7] S. G. Matthews, Partial Metric Topology, in: Papers on general topology and applications, Ann. New York Acad. Sci. 728 (1994), 183-197. 1, 1, 1.4

[8] Z. Mustafa, M. Arshad, S. U. Khan, J. Ahmad, M. M. M. Jaradat, Common fixed point for multivalued mappings in G-metric spaces with applications, J. Nonlinear Sci. Appl., 10 (2017), 2550-2564. 3

[9] D. Wardowski, Fixed points of a new type of contractive mappings in complete metric spaces, Fixed Point Theory Appl., 2012 (2012), 6 pages. 3, 3

[10] D. Wardowski, N. Van Dung, Fixed points of F-weak contractions on complete metric space, Demonstr. Math., 47 (2014), 146-155. 3 